JOURNAL OF SYNCHROTRON RADIATION

ISSN 1600-5775

Received 30 April 2020

Accepted 11 June 2020

Edited by A. Momose, Tohoku University, Japan

‡ Present address: MAX IV Laboratory, Lund University, 22100 Lund, Sweden.

$\S$ Present address: Division of Synchrotron Radiation Research, Department of Physics, Lund University, 22100 Lund, Sweden.

Keywords: refractive $\mathrm{X}$-ray optics; aberration correction; ptychography; phase plate.

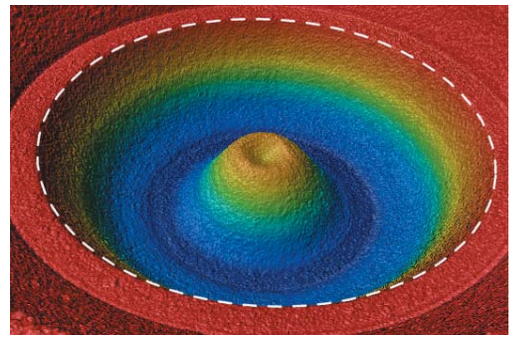

\section{Hard X-ray wavefront correction via refractive phase plates made by additive and subtractive fabrication techniques}

\author{
Frank Seiboth, ${ }^{\mathrm{a} *}$ Dennis Brückner, ${ }^{\mathrm{a}, \mathrm{b}, \mathrm{c}}$ Maik Kahnt, ${ }^{\mathrm{a}, \mathrm{b}} \neq$ Mikhail Lyubomirskiy, ${ }^{\mathrm{a}}$ \\ Felix Wittwer, ${ }^{\mathrm{a}, \mathrm{b}}$ Dmitry Dzhigaev, ${ }^{\mathrm{a}} \S$ Tobias Ullsperger, ${ }^{\mathrm{d}}$ Stefan Nolte, ${ }^{\mathrm{d}, \mathrm{e}}$ \\ Frieder Koch, ${ }^{f}$ Christian David, ${ }^{f}$ Jan Garrevoet, ${ }^{a}$ Gerald Falkenberg ${ }^{a}$ and \\ Christian G. Schroer ${ }^{\mathrm{a}, \mathrm{b}}$
}

a'Deutsches Elektronen-Synchrotron DESY, Notkestrasse 85, 22607 Hamburg, Germany, b Department Physik, Universität Hamburg, Luruper Chaussee 149, 22761 Hamburg, Germany, ${ }^{\mathrm{c}}$ Ruhr-Universität Bochum, Universitätsstrasse 150, 44801 Bochum, Germany, 'Institute of Applied Physics, Abbe Center of Photonics, Friedrich-Schiller-Universität Jena, Albert-Einstein-Strasse 15, 07745 Jena, Germany, ${ }^{\mathbf{e}}$ Fraunhofer Institute for Applied Optics and Precision Engineering, Albert-Einstein-Strasse 7, 07745 Jena, Germany, and 'Laboratory for Micro- and Nanotechnology, Paul Scherrer Institute, 5232 Villigen PSI, Switzerland. *Correspondence e-mail: frank.seiboth@desy.de

Modern subtractive and additive manufacturing techniques present new avenues for X-ray optics with complex shapes and patterns. Refractive phase plates acting as glasses for X-ray optics have been fabricated, and spherical aberration in refractive X-ray lenses made from beryllium has been successfully corrected. A diamond phase plate made by femtosecond laser ablation was found to improve the Strehl ratio of a lens stack with a numerical aperture (NA) of $0.88 \times 10^{-3}$ at $8.2 \mathrm{keV}$ from 0.1 to 0.7 . A polymer phase plate made by additive printing achieved an increase in the Strehl ratio of a lens stack at $35 \mathrm{keV}$ with NA of $0.18 \times 10^{-3}$ from 0.15 to 0.89 , demonstrating diffractionlimited nanofocusing at high $\mathrm{X}$-ray energies.

\section{Introduction}

An increasing number of ultra-low-emittance storage-ring sources are coming into operation (Tavares et al., 2018; Raimondi, 2016; Liu et al., 2014) and more facility upgrades and new facilities are planned (Hettel, 2014; Streun et al., 2018; Schroer et al., 2018; Jiao et al., 2018). The increase in brightness as a result of the strongly reduced source size and divergence will foster new science, if X-ray optics can preserve the beam properties. Due to higher lateral coherence, a larger portion of the X-ray beam can be used for diffraction-limited focusing. However, this requires optics with a sufficiently large aperture to collect the coherent beam. At the same time a high shape fidelity is required for diffraction-limited focusing. A similar challenge arises at X-ray free-electron lasers (XFELs), where ultrashort pulses and extremely high X-ray intensities are utilized, which puts the radiation resistance of current optics to the test (Uhlén et al., 2013; Koyama et al., 2016).

Parabolic compound refractive X-ray lenses (CRLs; Lengeler et al., 1999) represent one possible solution for those problems, as they have a large aperture, high radiation resistance, and diffraction-limited focusing capabilities well below $100 \mathrm{~nm}$. Over the past decades mechanically pressed beryllium (Be) lenses have been widely employed for beam transport and conditioning (Heimann et al., 2016), but also for nanofocusing at XFEL sources (Schropp et al., 2013; Seiboth et al., 2014b). Technical advances have enabled the microfabrication of diamond lenses by ion/plasma etching (Alianelli et al., 2010; 
Lyubomirskiy et al., 2019a), laser ablation (Terentyev et al., 2017; Antipov et al., 2018), or focused ion beam milling (Medvedskaya et al., 2020). Another new approach is the additive manufacturing of lenses by two-photon polymerization (Petrov et al., 2017; Lyubomirskiy et al., 2019b). So far, all techniques struggle with inherent limitations of the fabrication process and the production of X-ray optics with diffractionlimited performance, high numerical aperture (NA), large geometrical aperture, and sufficient radiation resistance is non-trivial. However, a constant evolution of these techniques offers not only the capability to fabricate lenses but also the freedom to produce almost any shape. Thus, new refractive optical elements for aberration correction (Sawhney et al., 2016; Seiboth et al., 2017; Laundy et al., 2019) and wavefront manipulation (Seiboth et al., 2019) have emerged. They provide an alternative to deformable mirrors (Mimura et al., 2010) and differential deposition methods (Matsuyama et al., 2018) for wavefront correction as well as to diffractive elements (Vila-Comamala et al., 2014; Loetgering et al., 2020) for wavefront manipulation.

Here, we investigate two possibilities to fabricate glasses for X-ray optics: Femtosecond laser ablation in diamond and printing by two-photon absorption in a polymer. Compared with our previous work the transition from ablation in fused silica (Seiboth et al., 2017) to diamond will enhance phase plate transmission and reduce the aspect ratio due to superior material properties of diamond. While polymer printing already showed excellent results in the past (Schropp et al., 2018 , 2019), the potential of this fabrication technique is further explored by printing phase plates with high aspect ratios that are required for aberration correction at high X-ray energies or to compensate for very strong wavefield deformation. Shape accuracy and surface properties of the fabricated phase plates are discussed, as well as their potential radiation resistance. The phase plates are then employed to correct spherical aberration of Be CRLs in two different nanofocusing scenarios. At a lower X-ray energy of $8.2 \mathrm{keV}$ we demonstrate focusing of X-rays by a lens with a NA of $0.88 \times 10^{-3}$. At a high X-ray energy of $35 \mathrm{keV}$ we achieve sub$100 \mathrm{~nm}$ focusing by a lens with a NA of $0.18 \times 10^{-3}$, giving a perspective for possible applications at fourth-generation sources with ultra-low emittance.

\section{Experimental setup}

To measure the focusing properties of the X-ray optics, we use ptychography (Thibault et al., 2008; Maiden \& Rodenburg, 2009), a scanning coherent diffraction imaging method used routinely to retrieve the complex wavefield for optics characterization (Schropp et al., 2010; Kewish et al., 2010a,b; VilaComamala et al., 2011; Hönig et al., 2011; Schropp et al., 2013; Uhlén et al., 2014; Seiboth et al., 2014a; Kubec et al., 2014; Morgan et al., 2015; Patommel et al., 2017). In particular, the performance of Be CRLs can be determined quantitatively. Ptychography works best if source size effects can be neglected, i.e. the X-ray beam illuminating the lens aperture is laterally coherent. In this case, the focus is dominated by diffraction. Its size and shape depends on the NA of the lens and any aberrations of the optics, rather than on the demagnified X-ray source size. Following Schroer \& Falkenberg (2014), the regime of diffraction-limited focusing of a refractive lens is reached when the effective aperture $D_{\text {eff }}$ of the optics is smaller than the lateral coherence length,

$$
l_{c_{h, v}}=\frac{2 \sqrt{2 \ln 2}}{\pi} \frac{\lambda L}{S_{\{h, v\}}}>D_{\text {eff }},
$$

where $\lambda$ is the $\mathrm{X}$-ray wavelength, $L$ is the source-to-optics distance and $S_{\{h, v\}}$ is the full width at half-maximum (FWHM) source size in the horizontal and vertical direction, respectively. In the following, the coherence properties are estimated for the given experimental conditions.

The Be CRLs used in these experiments were manufactured by RXOPTICS from beryllium IF-1 foils (Materion) with $0.5 \mathrm{~mm}$ thickness. The radius of curvature for these bi-concave lenses measures $50 \mu \mathrm{m}$ and their geometrical aperture $D=$ $300 \mu \mathrm{m}$. The effective aperture $D_{\text {eff }}$ is reduced compared with the geometrical aperture $D$ by the attenuation inside the lens material (Lengeler et al., 1999; Schroer \& Falkenberg, 2014) and depends on the particular experimental conditions.

For the experiments at $8.2 \mathrm{keV}$ a stack of $N=50$ lenses was used. It has an effective aperture of $D_{\text {eff }}=191 \mu \mathrm{m}$. For the experiments at $35 \mathrm{keV}$ the lens stack comprised $N=149$ lenses with an effective aperture of $D_{\text {eff }}=236 \mu \mathrm{m}$.

\subsection{Nanofocusing at $8.2 \mathrm{keV}$}

Experiments were carried out at the coherence branch I13-1 (Rau et al., 2011) of Diamond Light Source (DLS) at a photon energy of $8.2 \mathrm{keV}$. A sketch of the beamline layout can be seen in Fig. 1 and relevant distances are listed in Table 1. The FWHM X-ray source size created by the undulator measures $400 \mu \mathrm{m} \times 13 \mu \mathrm{m}(\mathrm{h} \times \mathrm{v})$. The vertical source is located in the center of the undulator; the horizontal source has its minimum size $11 \mathrm{~m}$ further downstream, which is $212 \mathrm{~m}$ upstream of the Be CRLs. According to equation (1) the lateral coherence length $l_{c_{h, v}}$ at the lens aperture is $60 \mu \mathrm{m} \times 1942 \mu \mathrm{m}(\mathrm{h} \times \mathrm{v})$. In order to satisfy $l_{\mathrm{c}_{h}}>D_{\text {eff }}=191 \mu \mathrm{m}$ we closed horizontal slits at $P_{\text {slit }}=20 \mathrm{~m}$ downstream of the undulator to $30 \mu \mathrm{m}$, increasing the lateral coherence length at the Be CRLs to $l_{c_{h}}=766 \mu \mathrm{m}$. $\mathrm{X}$-rays were focused by the stack of $50 \mathrm{Be}$ CRLs with a total length of $L_{\mathrm{CRL}}=55 \mathrm{~mm}$, resulting in a focal length of $108.6 \mathrm{~mm}$ and NA of $0.88 \times 10^{-3}$. The difference in the horizontal and vertical source position leads to astigmatic focusing. In the given geometry, however, the focal positions for the vertical and horizontal directions differ by less than the depth of focus. The effect is thus negligible.

The sample, a Siemens star pattern made out of $500 \mathrm{~nm}$ thick gold on a $200 \mathrm{~nm}$ silicon nitride support with $50 \mathrm{~nm}$ smallest features, was placed roughly $1 \mathrm{~mm}$ out of focus. We scanned the sample with a step size of $1 \mu \mathrm{m}$ across the beam, covering an area of $9 \mu \mathrm{m} \times 9 \mu \mathrm{m}$ with $10 \times 10$ scan points. Farfield diffraction patterns with a dwell time of $1 \mathrm{~s}$ were recorded with a quad chip Merlin photon-counting pixel detector (Plackett et al., 2013) at a distance of $3.675 \mathrm{~m}$ downstream of 
Table 1

Position of relevant experimental components along the beamlines I13-1 of DLS $(8.2 \mathrm{keV})$ and P06 of PETRA III $(35 \mathrm{keV})$; the corresponding sketch of the setup can be seen in Fig. 1.

\begin{tabular}{llccrrr}
\hline & & \multicolumn{2}{c}{ Position $(\mathrm{m}) \dagger$} & & \multicolumn{2}{c}{ Distance $(\mathrm{m}) \ddagger$} \\
Component & Symbol & I13-1 & P06 & & I13-1 & P06 \\
\hline Horizontal slits & $P_{\text {slit }}$ & 20 & 27 & & \\
Monochromator & $P_{\text {mono }}$ & 210 & 38 & & \\
Be CRL stack & $P_{\mathrm{CRL}} / D_{\mathrm{CRL}}$ & 223 & 93 & & -0.107 & -0.652 \\
Phase plate & $D_{\mathrm{Cpp}}$ & & & & -0.069 & -0.483 \\
Detector & $D_{\mathrm{det}}$ & & & & 3.675 & 8.435 \\
\hline
\end{tabular}

$\dagger$ Position along beamline from undulator source. $\$$ Relative distance to focal plane (cf. Fig. 1)

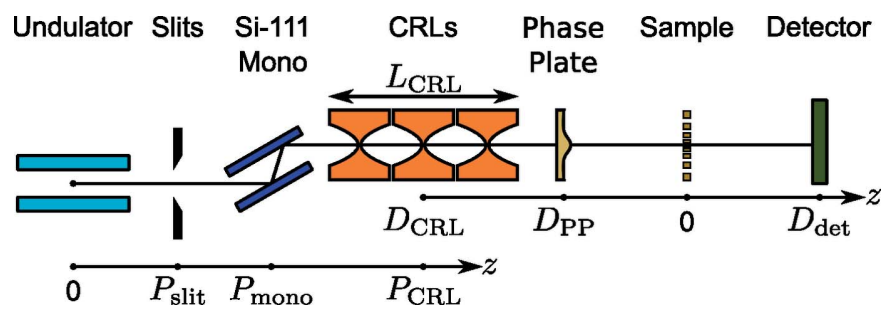

Figure 1

Schematic of the beamline setup at both I13-1 of DLS and P06 of PETRA III. At I13-1 we used a stack of $50 \mathrm{Be}$ CRLs at $8.2 \mathrm{keV}$ with a total length of $L_{\mathrm{CRL}}=55 \mathrm{~mm}$. At P06 we used 149 Be CRLs at $35 \mathrm{keV}$ with a stack length of $L_{\mathrm{CRL}}=298 \mathrm{~mm}$. All other distances can be found in Table 1.

the sample. The detector images were cropped to $446 \times 431$ pixels and zero-padded to $512 \times 512$ pixels, so that the optical axis is centered. With a detector pixel size of $55 \mu \mathrm{m}$ the resulting pixel size in the reconstructed images is $19.7 \mathrm{~nm}$. An example of the reconstructed object phase shift and complex probe field at the object position is shown in Figs. 2(a) and $2(b)$, respectively. From the reconstructed object shown in Fig. 2(a) one can clearly see the impact of mechanical instabilities in the horizontal direction, leading to a degrada-
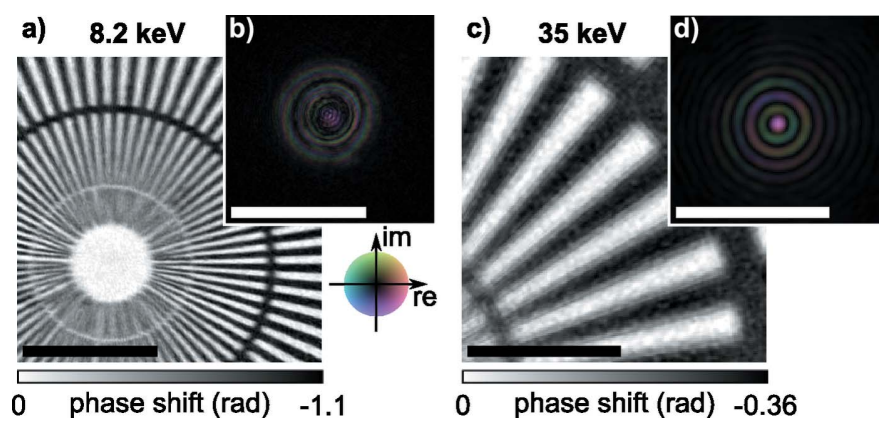

Figure 2

Ptychographic reconstructions of the test objects and illuminating wavefields obtained at $8.2 \mathrm{keV}(a, b)$ and $35 \mathrm{keV}(c, d)$. (a) Reconstructed object phase shift of a Siemens star patterned into a $500 \mathrm{~nm}$-thick gold layer. (b) Reconstructed complex illumination function in the object plane. Both images in $(a)$ and $(b)$ are shown at the same scale and the bar represents $4 \mu \mathrm{m}$. (c) Reconstructed object phase shift of a Siemens star patterned into a $500 \mathrm{~nm}$-thick tantalum layer. $(d)$ Reconstructed complex illumination function in the object plane. Both images in $(c)$ and $(d)$ are shown at the same scale and the bar represents $2 \mu \mathrm{m}$. tion in contrast for the vertically oriented spokes of the Siemens star.

\subsection{High-energy nanofocusing at $35 \mathrm{keV}$}

Measurements were carried out at the microprobe endstation of beamline P06 of PETRA III at DESY at a photon energy of $35 \mathrm{keV}$. A sketch of the beamline layout can be seen in Fig. 1 and relevant distances are listed in Table 1. Here, the FWHM X-ray source size measures $85 \mu \mathrm{m} \times 14 \mu \mathrm{m}(\mathrm{h} \times \mathrm{v})$ and is located $93 \mathrm{~m}$ upstream of the Be CRLs. According to equation (1) the lateral coherence length $l_{c_{h, v}}$ at the focusing optics measures $29 \mu \mathrm{m} \times 176 \mu \mathrm{m}(\mathrm{h} \times \mathrm{v})$. To increase the horizontal coherence length we closed horizontal slits at $P_{\text {slit }}=$ $27 \mathrm{~m}$ downstream of the undulator to $16 \mu \mathrm{m}$, yielding a lateral horizontal coherence length of $l_{c_{h}}=109 \mu \mathrm{m}$ at the Be CRLs. Thus, at these high X-ray energies, the effective aperture $D_{\text {eff }}=$ $236 \mu \mathrm{m}$ was not illuminated coherently.

X-rays were focused by a stack of 149 Be CRLs with a total length of $L_{\mathrm{CRL}}=298 \mathrm{~mm}$, resulting in a focal length of $653.6 \mathrm{~mm}$ and NA of $0.18 \times 10^{-3}$. A Siemens star test object made by NTT-AT (ATN/XRESO-50HC) with a $500 \mathrm{~nm}$-thick tungsten layer and $50 \mathrm{~nm}$ smallest features was placed roughly $3 \mathrm{~mm}$ out of focus. The object was scanned with a step size of $200 \mathrm{~nm}$ and $400 \mathrm{~nm}$, covering an area of $4 \mu \mathrm{m} \times 4 \mu \mathrm{m}$ with $20 \times 20$ and $10 \times 10$ steps, respectively. An X-Spectrum Lambda 2M detector (Pennicard et al., 2013) with a $500 \mu \mathrm{m}$ GaAs sensor was used to record far-field diffraction patterns at a distance of $8.435 \mathrm{~m}$ downstream of the sample. With a cropping of $256 \times 256$ pixels around the optical axis and a detector pixel size of $55 \mu \mathrm{m}$, the pixel size in the reconstructed images equals $21.2 \mathrm{~nm}$. An example of the reconstructed object phase shift and complex probe field at the object position is shown in Figs. $2(c)$ and $2(d)$, respectively. The reconstruction of the object is in part influenced by the reduced lateral coherence. However, main artifacts like the grainy background in Fig. 2(c) originate from the inhomogeneous response of the GaAs sensor.

\subsection{GaAs sensor inhomogeneity}

In Figs. 3(a) and 3(b) a typical scattering pattern for the Merlin as well as for the Lambda detector at $8.2 \mathrm{keV}$ and $35.0 \mathrm{keV}$ is shown, respectively. An average background image is calculated from the difference between measured diffraction patterns and those modeled based on the ptychographic reconstruction (Bernert et al., 2017). The resulting background that corresponds to the reconstructions shown in Fig. 2 is shown in Figs. 3(c) and 3(d) for the Merlin detector at $8.2 \mathrm{keV}$ and for the Lambda detector at $35.0 \mathrm{keV}$, respectively. While the Merlin background in Fig. 3(c) mainly shows incoherent scattering, the Lambda background in Fig. 3(d) shows a clear pattern originating from inhomogeneities in the GaAs sensor material. Although a flat-field correction was applied to the diffraction patterns, the large variations in the sensitivity of the sensor locally distort the diffraction patterns at low count rates. In addition to the reduced coherence at higher $\mathrm{X}$-ray energies, these features of the sensor material hamper 

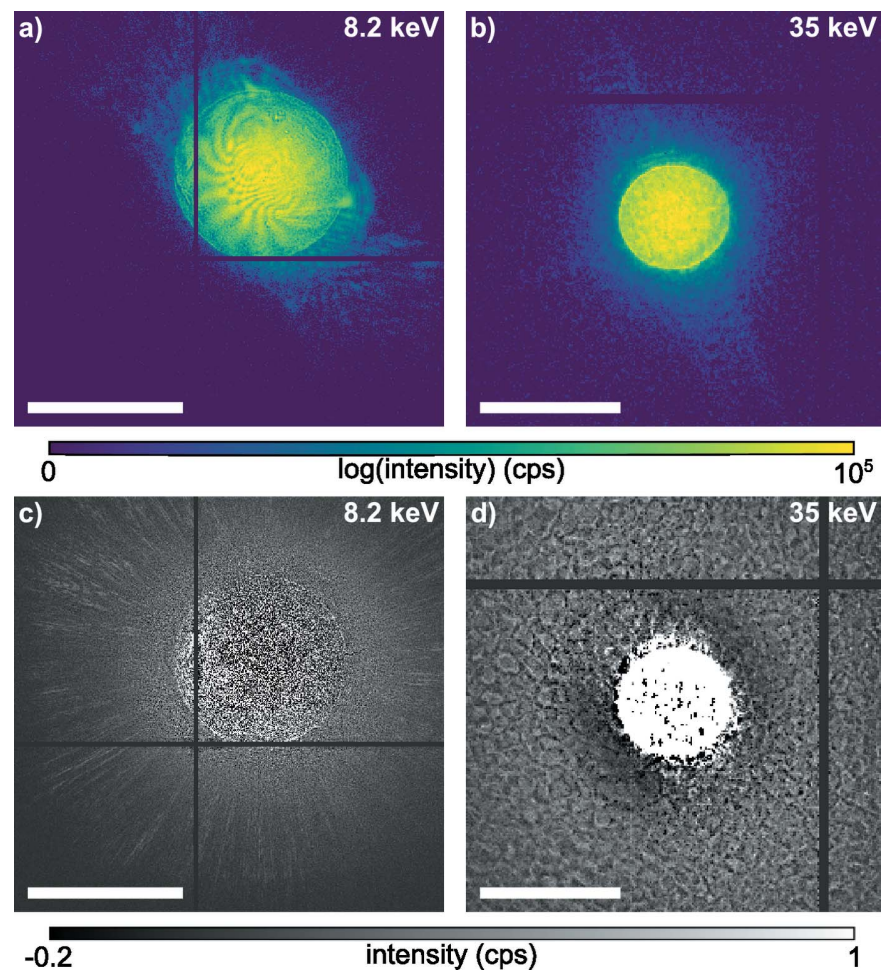

Figure 3

Example of diffraction patterns and average background signal, which is calculated from the difference between measured data and modeled diffraction patterns from ptychography. (a) Cropped diffraction pattern recorded with a Merlin detector at $8.2 \mathrm{keV}$. The beam is off-center as the detector ends in the upper right corner. (b) Cropped diffraction pattern at $35.0 \mathrm{keV}$ recorded with the Lambda $2 \mathrm{M}$ GaAs detector. (c) Average background at $8.2 \mathrm{keV}$ with the Merlin $\mathrm{Si}$ sensor. (d) Average background at $35 \mathrm{keV}$ with the Lambda GaAs sensor. The scale bar in all images represents a scattering vector of $0.1 \mathrm{~nm}^{-1}$.

the convergence of the ptychographic algorithm, leading to stronger reconstruction artifacts in the object as shown in Fig. 2(c). As varying sensitivity effects are more dominant for lower count rates at high scattering angles, reconstruction artifacts are mainly at high spatial frequency. As the probe, shown in Fig. $2(d)$, is kept constant within the ptychographic algorithm across all scan positions (Maiden \& Rodenburg, 2009), inconsistencies are pushed into the object and the probe wavefield is nevertheless reconstructed with confidence.

\section{Refractive phase plates}

From the initial wavefield characterization via ptychography, as shown in Fig. 2, one can numerically propagate the complex wavefield along the optical axis using the Fresnel-Kirchhoff diffraction integral. In Figs. $4(a)$ and $4(c)$ the beam caustic in the horizontal direction is shown for $8.2 \mathrm{keV}$ and $35 \mathrm{keV}$, respectively. The caustic is created by projecting the intensity of the three-dimensional wavefield of the beam onto the horizontal plane. A dashed line in Fig. 4(a) and 4(c) marks the plane with the highest peak intensity, which was assumed to be the principal focal plane. It is shown in Figs. $4(b)$ and $4(d)$ for $8.2 \mathrm{keV}$ and $35 \mathrm{keV}$, respectively. The beam caustics in Figs. 4(a) and 4(c) show areas further upstream with high

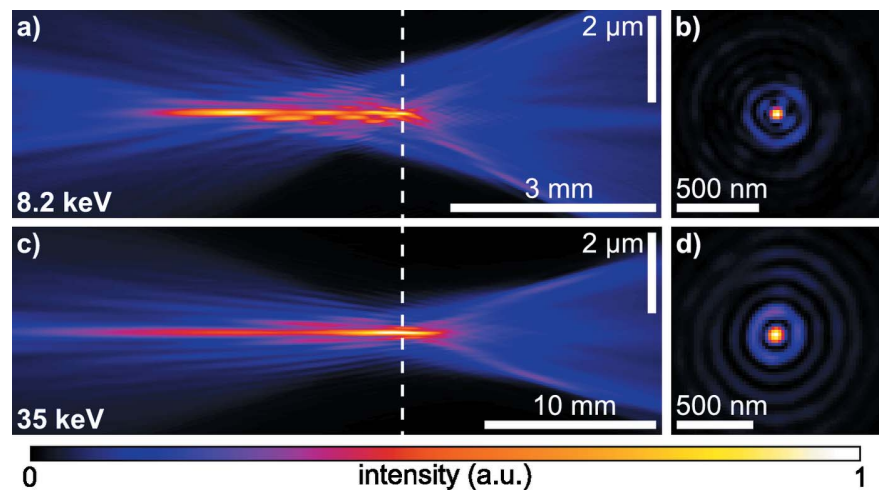

Figure 4

Characterized X-ray beams with spherical aberration. (a) Horizontal beam caustic at $8.2 \mathrm{keV}$. (b) Intensity distribution in the plane with highest peak intensity, marked by the dashed line in $(a)$. (c) Horizontal beam caustic at $35 \mathrm{keV}$. (d) Intensity distribution in the plane with highest peak intensity, marked by the dashed line in $(c)$.

intensity on the optical axis. These secondary focal planes originate from a varying curvature of the Be CRL along its rotational parabolic profile. More specifically, the inner part close to the optical axis of the Be CRL seems to be more strongly curved, leading to a shorter focal length for X-rays impinging close to the optical axis on the lens aperture (Schropp et al., 2013). This spherical aberration does not lead to a broadening of the focal spot, but rather creates strong side lobes around the central speckle, as shown in Figs. 4(b) and $4(d)$. To correct for these aberrations, which are caused by peak-to-valley (PV) shape inaccuracies of roughly $0.5 \mu \mathrm{m}$ in the printing tools used during lens manufacturing (Seiboth et al., 2017), we pursued the concept of an additional optical element to correct for any lens aberrations. Here, we use a phase plate based on refraction (Seiboth et al., 2017), similar to glasses for the correction of eyesight. An ideal focusing lens creates a converging spherical wave at its exit. Aberrations lead to a deformation of this ideal phase profile. The wavefield $\Psi_{\Delta z}(x, y)$ at any plane behind the lens can be determined from the measured complex illumination function via ptychography [see Figs. 2(b) and 2(d)] and by backpropagation of a distance $\Delta z$ along the optical axis. Subtracting a spherical wave $\varphi_{r}(x, y)=-k\left[\left(r^{2}+x^{2}+y^{2}\right)^{1 / 2}-r\right]$ with radius $r$ and wavenumber $k=2 \pi / \lambda$, which is fitted to the wavefront curvature, yields the residual wavefront error,

$$
\Psi_{\epsilon}=\Psi_{\Delta z}(x, y) \exp \left[i \varphi_{r}(x, y)\right] .
$$

The thickness profile $z_{\mathrm{pp}}(x, y)$ of the phase plate is designed to compensate any wavefront errors in $\Psi_{\epsilon}$ by introducing a phase shift $\varphi_{\mathrm{pp}}=-k \delta_{\mathrm{pp}}(k) z_{\mathrm{pp}}(x, y)$ via refraction, where $\delta_{\mathrm{pp}}(k)$ is the refractive index decrement of the phase plate material, so that

$$
\arg \left[\Psi_{\epsilon}(x, y)\right]=-\varphi_{\mathrm{pp}}(x, y) .
$$

The calculated phase plate profile $z_{\mathrm{pp}}(x, y)$ is only valid in the specific plane along the optical axis and at the measured photon energy. Since refractive power of the Be CRL changes with energy, the numerical aperture and convergence of the wavefield inside and after the Be CRL also changes. However, 
by translating the phase plate along the optical axis by a certain distance, one can compensate these effects and the phase plate can correct over a broad energy range (Seiboth $e t$ al., 2018). Instead of measuring the wavefield for a specific lens combination, one can also pursue the approach to characterize the thickness profile of individual lens elements (Celestre et al., 2020). This allows to numerically calculate any lens stack from the measured single lenses at arbitrary photon energies and to retrieve the potential wavefront deformation of this lens configuration numerically. Another approach for optical elements with very specific types of aberrations, like X-ray mirrors based on external total reflection, allows for the design of adaptable refractive elements to compensate the typical sinusoidal wavefront error found in these X-ray optics (Laundy et al., 2019).

\subsection{Diamond phase plates}

Diamond phase plates were manufactured from chemical vapour deposition (CVD)-grown single-crystal diamond substrates by Element Six measuring $2.6 \mathrm{~mm} \times 2.6 \mathrm{~mm} \times$ $0.3 \mathrm{~mm}$ with $\langle 100\rangle$ orientation. For ablation, we used an Amplitude Satsuma fiber laser with second-harmonic generation module. Pulses with a duration of $300 \mathrm{fs}$ and $515 \mathrm{~nm}$ wavelength were focused with a $20 \times$ objective (NA = $0.4)$ onto the substrate. To reduce absorption of the X-ray beam, the diamond substrate was thinned by $76 \mu \mathrm{m}$ in a first step with a laser repetition rate of $12 \mathrm{kHz}$ and pulse energy of $160 \mathrm{~nJ}$. Therefore, layers with a thickness of $0.5 \mu \mathrm{m}$ were sequentially ablated by scanning the focus across the sample surface with a hatch spacing of $0.5 \mu \mathrm{m}$ by moving the substrate and the objective with a high-precision three-axis motion system from Aerotech (ANT130 XY, LZ) with a scanning velocity of $0.3 \mathrm{~mm} \mathrm{~s}^{-1}$. Subsequently, the phase-plate structure was stepwise ablated with a repetition rate of $6 \mathrm{kHz}$ and pulse energy of $50 \mathrm{~nJ}$, similar to the procedure described by Seiboth et al. (2017). For this, the profile was sliced into 70 layers and the substrate was ablated only at locations determined by the design. A surface profile of the manufactured diamond phase plate, acquired by a Keyence VK-X1100 laserscanning microscope (LSM) with $50 \times$ objective and NA = 0.95 , is shown in Fig. 5(a). The radially averaged profile from LSM measurements is compared with the design goal in Fig. 5(b). The height profile from ptychography shown in Fig. 5(b) is calculated from the difference of the backpropagated wavefields without phase plate [see Fig. 6(a), left side] and with phase plate [see Fig. 6(a), right side]. Measured profiles agree well with each other and closely match the design goal to within $2 \mu \mathrm{m}$, as shown in the lower subplot of the error against the design goal for both measurements in Fig. 5(b). Due to the ablation process the surface is typically not smooth. A surface roughness of $s_{\mathrm{a}}=0.32 \mu \mathrm{m}$ was determined by LSM measurements.

\subsection{Polymer phase plates}

For the correction of 149 Be CRLs at $35 \mathrm{keV}$ a peak wavefront error of $9.5 \mathrm{rad}$ close to the optical axis had to be compensated. Due to very weak refraction at these high photon energies the aspect ratio of the resulting phase plate becomes critical for ablation techniques. Instead, we used additive manufacturing via two-photon polymerization in a Nanoscribe IP-S resist, as demonstrated by Schropp et al. (2018) and Seaberg et al. (2019). The structure was written
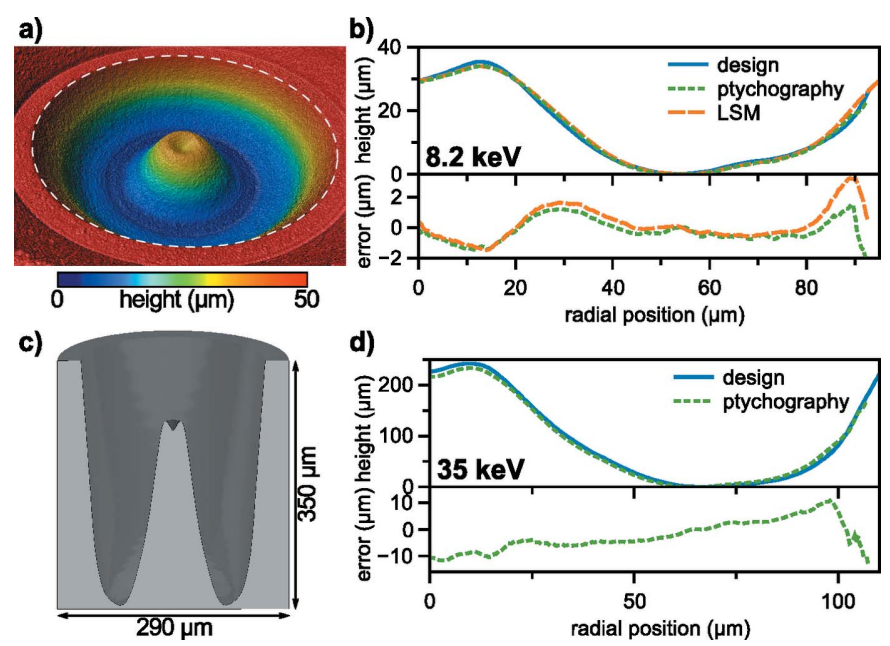

Figure 5

Refractive phase plates and their thickness profiles. (a) Surface of the diamond phase plate used at $8.2 \mathrm{keV}$, acquired by a Keyence VK-X1100 laser scanning microsope (LSM). The dashed circle represents a diameter of $220 \mu \mathrm{m}$. The optically relevant region of the phase plate has a smaller diameter of only $186 \mu \mathrm{m}$. (b) Radial height profiles of the diamond phase plate shown in $(a)$ measured via ptychography (green, dotted line) and LSM (orange, dashed line) in comparison with the design goal (blue, solid line). The error for both measurements against the design goal is shown in the lower subplot. (c) 3D rendering of the polymer phase plate used at $35 \mathrm{keV}$. The model is sliced in the middle for better visibility of the characteristic phase plate shape. $(d)$ Radial height profile of the polymer phase plate shown in (c) measured via ptychography (green, dotted line) in comparison with the design goal (blue, solid line). The error against the design goal is shown in the lower subplot.

Figure 6

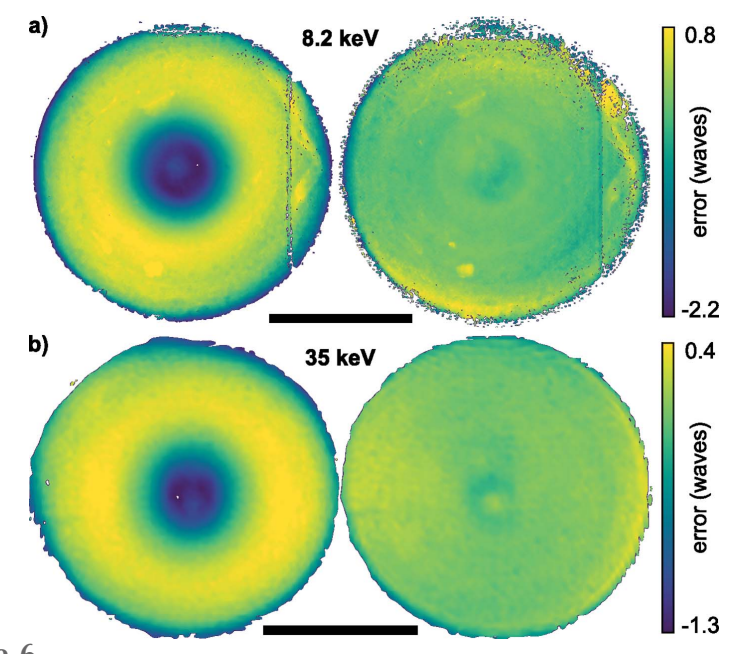

Residual wavefield error in the plane of the phase plate. (a) Wavefield error in the plane located $10.5 \mathrm{~mm}$ downstream of the lens exit aperture of $50 \mathrm{Be}$ CRLs at $8.2 \mathrm{keV}$ with (right) and without (left) corrective phase plate. (b) Wavefield error in the plane located $20 \mathrm{~mm}$ downstream of the lens exit aperture of $149 \mathrm{Be}$ CRL at $35 \mathrm{keV}$ with (right) and without (left) corrective phase plate. The scale bar in both figures represents $100 \mu \mathrm{m}$. 
with a Nanoscribe Photonic Professional GT in dip-in lithography mode with a $25 \times$ objective $(\mathrm{NA}=0.8)$. Slicing and hatching of the three-dimensional structure was performed with a spacing of $200 \mathrm{~nm}$. For fast lateral writing a galvo scanner system was used. Vertical displacement was achieved by a mechanical drive, as the piezo drive did not provide enough travel for the structure to be written without stitching. A three-dimensional model of the phase plate, which is sliced in the middle, is shown in Fig. 5(c). The outer ring with $350 \mu \mathrm{m}$ height is printed for alignment purposes only and is not optically relevant for aberration correction. In Fig. $5(d)$ the relevant radial profile for aberration correction is shown. As the whole shape profile of the polymer phase plate could not be measured accurately by the LSM, only a height reconstruction from ptychography is shown. A thin Au coating of the polymer structure could potentially improve the reflectivity and thus the measurement success by LSM, but the steep slope angles in this particular case are an additional challenge. Instead, the profile is retrieved by subtracting the wavefield in the plane of the phase plate from two different ptychography measurements shown in Fig. 6(b), as described earlier in Section 3.1. The shape agrees well with the design, but the height scale does not fully match: the difference in PV height is about $10 \mu \mathrm{m}$ [see the lower error plot in Fig. 5(d)] and corresponds to a $<5 \%$ systematic error. There are two potential influencing factors for this systematic error: a deviation of the height scale of the printed structures relative to the nominal ones and a deviation of the index of refraction decrement $\delta_{\mathrm{pp}}$ from the value used for the design. While the LSM could not follow the full profile on the steepest slopes it was possible to measure the flatter areas of the phase plate at the bottom and on the top of the central cone: at the radial position of $10 \mu \mathrm{m}$ on the central cone a height of $246 \mu \mathrm{m}$ was measured relative to the lowest points. This is to be compared with $242 \mu \mathrm{m}$ of the design and with $232 \mu \mathrm{m}$ calculated with the nominal $\delta_{\mathrm{pp}}$ from ptychography. This indicates that $\delta_{\mathrm{pp}}$ of the IP-S resist might be $2.124 \times 10^{-7}$ at $35 \mathrm{keV}$ instead of $2.216 \times 10^{-7}$ used for the modeling. Since there are no precise measurements of the polymer's refractive index in the X-ray regime, and both the density and mass proportions of the different photoresist constituents are not precisely known, an error in the estimated $\delta_{\mathrm{pp}}$ is in fact expected.

\section{Results}

Aberrated Be CRL stacks at both $8.2 \mathrm{keV}$ and $35 \mathrm{keV}$ were corrected by placing the respective phase plate directly behind the lens casing. As noted in Table 1 the phase plate was positioned at a distance of $D_{\mathrm{pp}}$ in front of the sample. Together with the position of the lens stack center $D_{\mathrm{CRL}}$ and the lens length $L_{\mathrm{CRL}}$ the phase plates were mounted $\left|D_{\mathrm{CRL}}\right|-\left|D_{\mathrm{Cpp}}\right|-$ $L_{\mathrm{CRL}} / 2=10.5 \mathrm{~mm}$ and $20 \mathrm{~mm}$ after the lens exit for $8.2 \mathrm{keV}$ and $35 \mathrm{keV}$, respectively. They were fixed along the optical axis and aligned perpendicular to the X-ray beam to within $2 \mu \mathrm{m}$. In a first step they were aligned to better than $5 \mu \mathrm{m}$ by eye with the help of a scintillator-based high-resolution X-ray microscope in transmission geometry. Afterwards, wavefront characterization via ptychography was carried out and the position of the phase plate adjusted by typically $2 \mu \mathrm{m}$ or less to reach best focus. Once aligned, the position was stable over several days and no further realignment was required.

\subsection{Focusing at $8.2 \mathrm{keV}$}

Before aberration correction, the wavefront error $10.5 \mathrm{~mm}$ downstream of the lens [left side in Fig. 6(a)] showed the typical rotational symmetry of spherical aberration with a PV error of $3.5 \lambda$ and root-mean-square (RMS) error of $0.75 \lambda$. After alignment of the diamond phase plate shown in Figs. 5(a) and $5(b)$, the wavefront distortion reduced to a PV error of $1.11 \lambda$ and RMS error of $0.26 \lambda$, shown on the right side in Fig. 6(a). Remaining errors are dominated by lens defects, visible as yellow spots in the lower part and on the right side of the wavefield in Fig. 6(a). The central area of the corrected wavefield indicates a slight shape mismatch of the phase plate compared with the design goal, as shown in Fig. 5(b). A vertical stripe on the right side of the lens aperture originates from the panel gap of the Merlin detector going through the direct beam in the diffraction patterns [see Fig. 3(a)]. The resulting beam caustic and focal spot of the corrected lens is shown in Figs. 7(a) and 7(b). X-rays are concentrated into a single focal plane marked by the dashed line, leading to a Gaussian focal spot with high intensity and reduced side lobes. The logarithmic plot in the upper part of Fig. 8(a) shows a suppression of side-lobe intensity between one and two orders of magnitude. The effect of side lobes and their impact on scanning microscopy techniques like fluorescence imaging becomes evident when comparing the radially integrated photon flux, shown in Fig. $8(b)$. Solid lines indicate the intensity distribution at $8.2 \mathrm{keV}$. While $\sim 75 \%$ of the intensity is homogeneously distributed over a radius of $986 \mathrm{~nm}$ for the aberrated lens (blue line), an improvement down to $138 \mathrm{~nm}$ with the phase plate (orange line) is observed. The theoretical value (green line) is at $52 \mathrm{~nm}$. The FWHM focal spot size
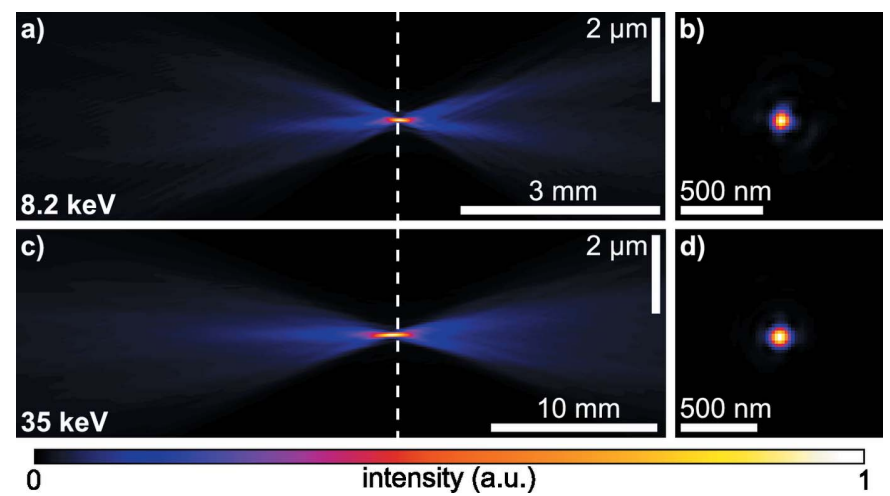

Figure 7

$\mathrm{X}$-ray beams after correction with a phase plate as characterized by ptychography. (a) Horizontal beam caustic at $8.2 \mathrm{keV}$ after correction with the diamond phase plate shown in Fig. 5(a). (b) Intensity distribution in the plane with highest peak intensity, marked by the dashed line in $(a)$. (c) Horizontal beam caustic at $35 \mathrm{keV}$ after correction with the polymer phase plate shown in Fig. 5(c). (d) Intensity distribution in the plane with highest peak intensity, marked by the dashed line in $(c)$. 

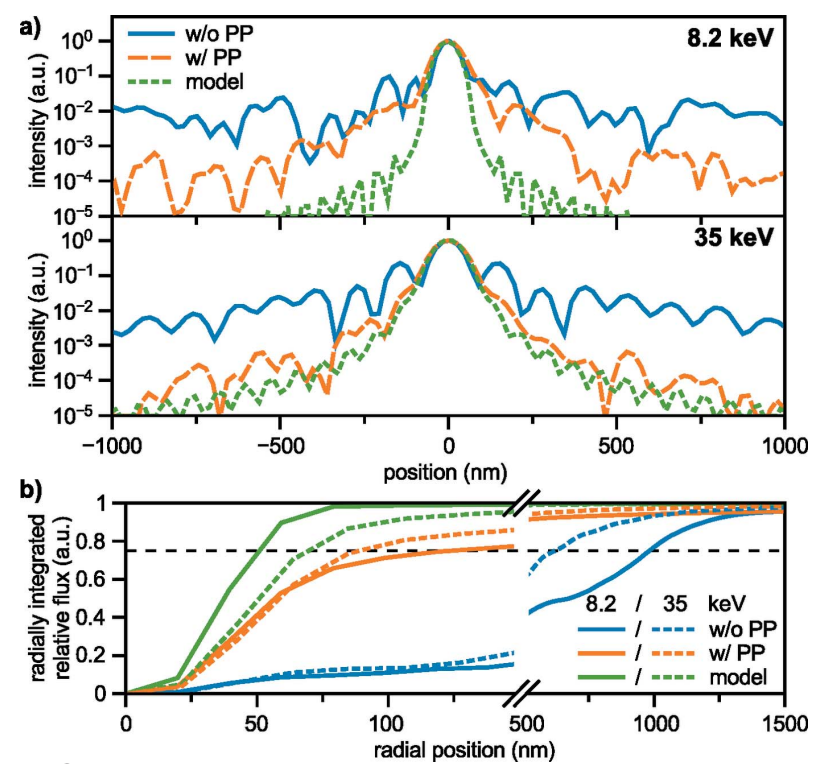

Figure 8

Intensity distribution in the focal plane. (a) Upper subplot: horizontal beam profile at $8.2 \mathrm{keV}$ with the diamond phase plate (orange, dashed line) compared with the aberrated (blue, solid line) and ideal (green, dotted line) lens. Lower subplot: horizontal beam profile at $35 \mathrm{keV}$ with the polymer phase plate (orange, dashed line) compared with the aberrated (blue, solid line) and ideal (green, dotted line) lens. (b) Radially integrated intensity distribution at both $8.2 \mathrm{keV}$ (solid lines) and $35 \mathrm{keV}$ (dotted lines), comparing the aberrated lens (blue) with the phase plate corrected (orange) and ideal (green) lens. The dashed horizontal line marks 0.75 .

changed from $69 \mathrm{~nm}$ without the phase plate to $76 \mathrm{~nm}$ with the diamond phase plate. In the aberrated case the focal length varies with radius over the lens aperture. The convergent rays from outer parts of the lens aperture are focused into the plane marked by the dashed line in Fig. 4(a). Rays passing the lens close to the optical axis are focused further upstream. Thus, only a ring-shaped outer part of the lens contributes to the focus in the dashed plane. This aperture shape influences the width of the central speckle. As only large angles or high spatial frequencies contribute, the central speckle size is decreased (Kiss, 2016), but the strength of side lobes is enhanced at the same time due to missing lower spatial frequencies. If the whole lens aperture contributes, as is the case for the aberration-corrected lens in Fig. 7(a), the size of the central speckle increases slightly, but side lobes are suppressed. The Strehl ratio, measured as integrated intensity in the central speckle, increased from 0.10 to 0.70 . As the diamond phase plate has a transmission of $75 \%$, the total transmission of the optical system changed from $16.5 \%$ down to $12.4 \%$. Focal spot parameters are also summarized in Table 2.

\subsection{High-energy nanofocusing}

The large lens stack at $35 \mathrm{keV}$ also shows the typical pattern of spherical aberration in a plane located $20 \mathrm{~mm}$ behind the lens exit [left side in Fig. 6(b)], but appears more homogeneous with no visible lens defects or dirt. The PV wavefront error is $1.7 \lambda$ and the RMS error $0.38 \lambda$. After aligning the
Table 2

Achieved focal spot size and Strehl ratio for the Be CRL and phase plate combinations investigated.

\begin{tabular}{llllll}
\hline Beamline & $\begin{array}{l}\text { Energy } \\
(\mathrm{keV})\end{array}$ & $\begin{array}{l}\text { Phase } \\
\text { plate }\end{array}$ & $\begin{array}{l}\text { Focus size } \\
\text { FWHM } \\
(\mathrm{nm})\end{array}$ & $\begin{array}{l}\text { Strehl } \\
\text { ratio } \dagger\end{array}$ & Transmission \\
\hline DLS I13-1 & 8.2 & - & 69 & 0.10 & 0.165 \\
DLS I13-1 & 8.2 & Diamond $\ddagger$ & 76 & 0.70 & 0.124 \\
PETRA III P06 & 35.0 & - & 85 & 0.15 & 0.356 \\
PETRA III P06 & 35.0 & Polymer§ & 95 & 0.89 & 0.352 \\
\hline
\end{tabular}

$\dagger$ Ratio of integrated intensity in central speckle compared to ideal, modeled lens. $\ddagger$ See Figs. 5(a) and 5(b). $\S$ See Figs. 5(c) and 5(d)

polymer phase plate [see Fig. 5(c)], the PV wavefront error reduced to $0.7 \lambda$ and the RMS value to $0.11 \lambda$. In the central part of the aperture the slight height deviation of the fabricated phase plate [see Fig. $5(d)$ ] is visible by the residual donut-shaped phase ring. A weak astigmatism, originating from the different source position in the horizontal and vertical direction due to the horizontal slits (see Fig. 1 and Table 1), can be seen by the slightly larger phase error in horizontal than in the vertical direction. The horizontal beam caustic after aberration correction is shown in Fig. 7(c). The dashed line marks the focal plane. Due to a slight astigmatism this plane falls between the best focus in the horizontal and vertical direction (separated by roughly $600 \mu \mathrm{m}$ ). Since the depth of focus is $700 \mu \mathrm{m}$, the influence on the focal spot size is small. A logarithmic plot of the focal spot profile in the horizontal direction is shown in the lower part of Fig. 8(a). Side lobes are suppressed by two orders of magnitude for the phase-plate-corrected lens (dashed, orange line) compared with the aberrated lens (blue, solid line) and follow closely the theoretical limit (green, dashed line). The influence of the radially integrated flux in focus is depicted in Fig. 8(b) by the dashed lines for $35 \mathrm{keV}$. Without a phase plate $\sim 75 \%$ of the radiation is spread over a radius of $637 \mathrm{~nm}$. The phase plate improves this value to $90 \mathrm{~nm}$ (orange, dashed line) compared with the theoretical limit of $78 \mathrm{~nm}$ (green, dashed line). An increase in FWHM spot size is observed from $85 \mathrm{~nm}$ to $95 \mathrm{~nm}$, mainly caused by astigmatism. The Strehl ratio improved from 0.15 to 0.89 , indicating a diffraction-limited performance. Since the polymer is highly transparent for X-rays (transmission $>99 \%$ ), the total lens transmission stays almost unchanged at $35 \%$. A summary of the values can be found in Table 2.

At high X-ray energies the lateral coherence of the storagering source decreases and diffraction-limited operation of large-aperture optics is challenging, as discussed in Section 2.2. Using spatial filtering, that is closing horizontal slits to $16 \mu \mathrm{m}$, the lateral coherence length is increased to enable ptychographic imaging. In this way, the shape of a coherent mode can still be retrieved from a wavefield reconstruction via ptychography [see Fig. 2(b)]. The real spot size is typically larger due to source effects. In Fig. 9(a) the reconstructed object of a Siemens star test sample (NTT-AT ATN/XRESO-50HC, see Section 2.2) is compared with the fluorescence signal in Fig. $9(b)$. The data were acquired during the same measure- 

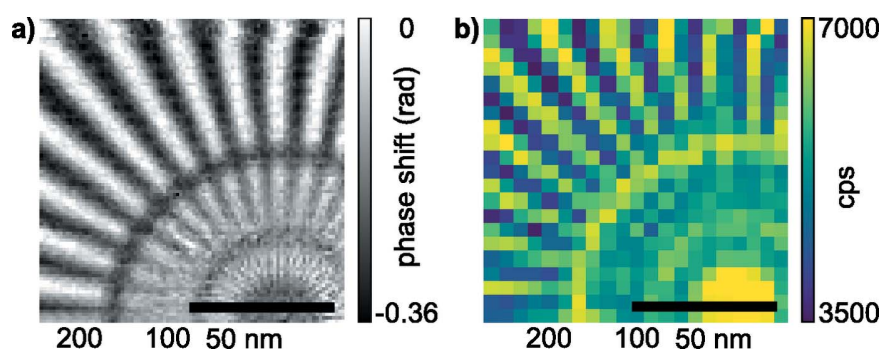

Figure 9

Resolution in scanning X-ray microscopy at $35 \mathrm{keV}$ with corrective phase plate. (a) Reconstructed object phase shift via ptychography from the central part of a Siemens star test object. Spoke features range from $50 \mathrm{~nm}$ size in the innermost circle to about $200 \mathrm{~nm}$ for the outermost circle. (b) The same area as in $(a)$, but in fluorescence contrast. The scan was performed with $100 \mathrm{~nm}$ steps with $21 \times 21$ scan points. The scale bar in both images represents $2 \mu \mathrm{m}$. The innermost spoke size in each radial segment is noted below the figures.

ment, collecting far-field diffraction patterns and fluorescence signal at once. The resolution of Fig. 9(a) depends on the scattering angle in the diffraction patterns and is independent of beam size. Although the reconstruction suffers from a reduced lateral coherence, sample instability, and detector inhomogeneity [see Fig. $3(d)$ ], the image provides a reference. The $100 \mathrm{~nm}$-sized spokes in the middle ring are clearly resolved, whereas the $50 \mathrm{~nm}$ features in the innermost ring are not resolved due to data quality. The fluorescence map shown in Fig. 9(b) gives a good measure for the incoherent beam size, including source-size effects. The image was acquired in $21 \times$ 21 steps with $100 \mathrm{~nm}$ step size. $200 \mathrm{~nm}$-sized outermost spokes are clearly resolved. A focal spot size of about $260 \mathrm{~nm}$ FWHM was determined from fluorescence knife-edges on a different area of the same sample.

\section{Conclusions}

We have demonstrated aberration correction of X-ray lenses via refractive phase plates made from two different materials and with complementary manufacturing techniques. In addition, aberration correction was successfully demonstrated not only for commonly used X-ray energies around $10 \mathrm{keV}$ but also for high-energy X-rays at $35 \mathrm{keV}$. A stack of 50 Be CRLs with a NA of $0.88 \times 10^{-3}$ at $8.2 \mathrm{keV}$ was corrected by a singlecrystal CVD-diamond phase plate fabricated via laser ablation. The Strehl ratio improved from 0.10 to 0.70 , while the total transmission of the optical system decreased by $25 \%$ due to absorption within the phase plate. The achieved aberrationcorrected focal spot size measures 76 nm FWHM. At $35 \mathrm{keV}$ we corrected a stack of 149 Be CRLs with a NA of $0.18 \times 10^{-3}$ by using a polymer phase plate manufactured additively via two-photon polymerization. The Strehl ratio improved from 0.15 to 0.89 , indicating a diffraction-limited performance. The coherent focal spot size, neglecting source effects, measures $95 \mathrm{~nm}$ FWHM. Fluorescence knife-edge scans indicate a focal spot size of $260 \mathrm{~nm}$, including source size effects.

Phase plates have become an important instrument to achieve better performance with refractive X-ray lenses at both storage-ring sources and XFELs. The two manufacturing approaches described here lead to complementary properties of optics. Laser ablation of diamond provides optics with a decent shape accuracy down to $1 \mu \mathrm{m}$, but the ablation process results in a large surface roughness. Subsequent etching or polishing (Antipov et al., 2018) might be suitable to improve surface roughness in the future. The advantage of diamond is its high thermal conductivity in combination with low absorption, which makes these optics a promising choice for high-intensity applications at XFELs. Recent developments in the fabrication of diamond micro-CRLs using ion beam lithography (Medvedskaya et al., 2020) could be a viable method to improve both shape accuracy and surface roughness for diamond optics.

Additive printing technology provides a high level of shape accuracy below $200 \mathrm{~nm}$ and very smooth surfaces. These optics are especially suited for storage-ring applications, where an operation over several weeks at beamline P06 showed a good radiation resistance. In addition, the material is highly transparent for X-rays. Whether the polymer phase plates can withstand several days of beam time at an XFEL remains an open question and might also strongly depend on the pulse train structure. At high X-ray energies above $20 \mathrm{keV}$ the spot size is often limited by the typical source properties of third-generation storage-ring facilities when working with large-aperture optics. Fourth-generation ultra-low-emittance storage ring sources and hard XFELs that operate well above $20 \mathrm{keV}$ in combination with aberration-corrected large-aperture optics will allow to collect a large fraction of the emitted light while efficiently focusing X-rays down to $100 \mathrm{~nm}$ and below, providing new opportunities for the investigation of thick samples and fluorescence imaging.

\section{Acknowledgements}

We acknowledge DESY (Hamburg, Germany), a member of the Helmholtz Association HGF, for the provision of experimental facilities. Parts of this research were carried out at beamline P06 of PETRA III. Parts of this research were carried out at the coherence branch of beamline I13-1 at Diamond Light Source (DLS) within experiment MT19906-1. This research was supported in part through the Maxwell computational resources operated at Deutsches ElektronenSynchrotron (DESY), Hamburg, Germany. The authors thank Darren Batey, Satishkumar Kulkarni, and Christoph Rau for support during beamtime at I13-1 and Ralph Döhrmann for engineering support. Open access funding enabled and organized by Projekt DEAL.

\section{Funding information}

Funding for this research was provided by: Volkswagen Foundation (scholarship to Frank Seiboth).

\section{References}

Alianelli, L., Sawhney, K. J. S., Malik, A., Fox, O. J. L., May, P. W., Stevens, R., Loader, I. M. \& Wilson, M. C. (2010). J. Appl. Phys. 108, 123107. 
Antipov, S., Assoufid, L., Grizolli, W., Qian, J. \& Shi, X. (2018). Proceedings of the 9th International Particle Accelerator Conference (IPAC'18), 29 April-4 May 2018, Vancouver, BC, Canada, pp. 4057-4058. THPMF011.

Bernert, C., Hoppe, R., Wittwer, F., Woike, T. \& Schroer, C. G. (2017). Opt. Express, 25, 31640.

Celestre, R., Berujon, S., Roth, T., Sanchez del Rio, M. \& Barrett, R. (2020). J. Synchrotron Rad. 27, 305-318.

Heimann, P., MacDonald, M., Nagler, B., Lee, H. J., Galtier, E., Arnold, B. \& Xing, Z. (2016). J. Synchrotron Rad. 23, 425-429.

Hettel, R. (2014). J. Synchrotron Rad. 21, 843-855.

Hönig, S., Hoppe, R., Patommel, J., Schropp, A., Stephan, S., Schöder, S., Burghammer, M. \& Schroer, C. G. (2011). Opt. Express, 19, 16325-16329.

Jiao, Y., Xu, G., Cui, X.-H., Duan, Z., Guo, Y.-Y., He, P., Ji, D.-H., Li, J.-Y., Li, X.-Y., Meng, C., Peng, Y.-M., Tian, S.-K., Wang, J.-Q., Wang, N., Wei, Y.-Y., Xu, H.-S., Yan, F., Yu, C.-H., Zhao, Y.-L. \& Qin, Q. (2018). J. Synchrotron Rad. 25, 1611-1618.

Kewish, C. M., Guizar-Sicairos, M., Liu, C., Qian, J., Shi, B., Benson, C., Khounsary, A. M., Vila-Comamala, J., Bunk, O., Fienup, J. R., Macrander, A. T. \& Assoufid, L. (2010a). Opt. Express, 18, 2342023427.

Kewish, C. M., Thibault, P., Dierolf, M., Bunk, O., Menzel, A., VilaComamala, J., Jefimovs, K. \& Pfeiffer, F. (2010b). Ultramicroscopy, 110, 325-329.

Kiss, M. Z. (2016). J. Eur. Opt. Soc.-Rapid Publ. 12, 2.

Koyama, T., Yumoto, H., Miura, T., Tono, K., Togashi, T., Inubushi, Y., Katayama, T., Kim, J., Matsuyama, S., Yabashi, M., Yamauchi, K. \& Ohashi, H. (2016). Rev. Sci. Instrum. 87, 051801.

Kubec, A., Braun, S., Niese, S., Krüger, P., Patommel, J., Hecker, M., Leson, A. \& Schroer, C. G. (2014). J. Synchrotron Rad. 21, 11221127.

Laundy, D., Dhamgaye, V., Moxham, T. \& Sawhney, K. (2019). Optica, 6, 1484-1490.

Lengeler, B., Schroer, C., Tümmler, J., Benner, B., Richwin, M., Snigirev, A., Snigireva, I. \& Drakopoulos, M. (1999). J. Synchrotron Rad. 6, 1153-1167.

Liu, L., Milas, N., Mukai, A. H. C., Resende, X. R. \& de Sá, F. H. (2014). J. Synchrotron Rad. 21, 904-911.

Loetgering, L., Baluktsian, M., Keskinbora, K., Horstmeyer, R., Wilhein, T., Schütz, G., Eikema, K. S. E. \& Witte, S. (2020). Sci. $A d v$. 6, eaax8836.

Lyubomirskiy, M., Boye, P., Feldkamp, J. M., Patommel, J., Schoeder, S., Schropp, A., Burghammer, M., Wild, C. \& Schroer, C. G. (2019a). J. Synchrotron Rad. 26, 1554-1557.

Lyubomirskiy, M., Koch, F., Abrashitova, K. A., Bessonov, V. O., Kokareva, N., Petrov, A., Seiboth, F., Wittwer, F., Kahnt, M., Seyrich, M., Fedyanin, A. A., David, C. \& Schroer, C. G. (2019b). Opt. Express, 27, 8639-8650.

Maiden, A. M. \& Rodenburg, J. M. (2009). Ultramicroscopy, 109, 1256-1262.

Matsuyama, S., Inoue, T., Yamada, J., Kim, J., Yumoto, H., Inubushi, Y., Osaka, T., Inoue, I., Koyama, T., Tono, K., Ohashi, H., Yabashi, M., Ishikawa, T. \& Yamauchi, K. (2018). Sci. Rep. 8, 17440.

Medvedskaya, P., Lyatun, I., Shevyrtalov, S., Polikarpov, M., Snigireva, I., Yunkin, V. \& Snigirev, A. (2020). Opt. Express, 28, 4773-4785.

Mimura, H., Handa, S., Kimura, T., Yumoto, H., Yamakawa, D., Yokoyama, H., Matsuyama, S., Inagaki, K., Yamamura, K., Sano, Y., Tamasaku, K., Nishino, Y., Yabashi, M., Ishikawa, T. \& Yamauchi, K. (2010). Nat. Phys. 6, 122-125.

Morgan, A. J., Prasciolu, M., Andrejczuk, A., Krzywinski, J., Meents, A., Pennicard, D., Graafsma, H., Barty, A., Bean, R. J., Barthelmess, M., Oberthuer, D., Yefanov, O., Aquila, A., Chapman, H. N. \& Bajt, S. (2015). Sci. Rep. 5, 9892.

Patommel, J., Klare, S., Hoppe, R., Ritter, S., Samberg, D., Wittwer, F., Jahn, A., Richter, K., Wenzel, C., Bartha, J. W., Scholz, M., Seiboth,
F., Boesenberg, U., Falkenberg, G. \& Schroer, C. G. (2017). Appl. Phys. Lett. 110, 101103.

Pennicard, D., Lange, S., Smoljanin, S., Hirsemann, H., Graafsma, H., Epple, M., Zuvic, M., Lampert, M.-O., Fritzsch, T. \& Rothermund, M. (2013). J. Phys. Conf. Ser. 425, 062010.

Petrov, A. K., Bessonov, V. O., Abrashitova, K. A., Kokareva, N. G., Safronov, K. R., Barannikov, A. A., Ershov, P. A., Klimova, N. B., Lyatun, I. I., Yunkin, V. A., Polikarpov, M., Snigireva, I., Fedyanin, A. A. \& Snigirev, A. (2017). Opt. Express, 25, 1417314181.

Plackett, R., Horswell, I., Gimenez, E. N., Marchal, J., Omar, D. \& Tartoni, N. (2013). J. Instrum. 8, C01038.

Raimondi, P. (2016). Synchrotron Radiat. News, 29(6), 8-15.

Rau, C., Wagner, U., Pešić, Z. \& De Fanis, A. (2011). Phys. Status Solidi A, 208, 2522-2525.

Sawhney, K., Laundy, D., Dhamgaye, V. \& Pape, I. (2016). Appl. Phys. Lett. 109, 051904.

Schroer, C. G., Agapov, I., Brefeld, W., Brinkmann, R., Chae, Y.-C., Chao, H.-C., Eriksson, M., Keil, J., Nuel Gavaldà, X., Röhlsberger, R., Seeck, O. H., Sprung, M., Tischer, M., Wanzenberg, R. \& Weckert, E. (2018). J. Synchrotron Rad. 25, $1277-$ 1290.

Schroer, C. G. \& Falkenberg, G. (2014). J. Synchrotron Rad. 21, 9961005.

Schropp, A., Boye, P., Feldkamp, J. M., Hoppe, R., Patommel, J., Samberg, D., Stephan, S., Giewekemeyer, K., Wilke, R. N., Salditt, T., Gulden, J., Mancuso, A. P., Vartanyants, I. A., Weckert, E., Schöder, S., Burghammer, M. \& Schroer, C. G. (2010). Appl. Phys. Lett. 96, 091102.

Schropp, A., Brückner, D., Bulda, J., Falkenberg, G., Garrevoet, J., Hagemann, J., Seiboth, F., Spiers, K., Koch, F., David, C., Gambino, M., Veselý, M., Meirer, F. \& Schroer, C. G. (2019). Proc. SPIE, 11112, 20-26.

Schropp, A., Brückner, D., Bulda, J., Falkenberg, G., Garrevoet, J., Seiboth, F., Wittwer, F., Koch, F., David, C., Schroer, C. G. (2018). Microsc. Microanal. 24(Suppl. 2), 186-187.

Schropp, A., Hoppe, R., Meier, V., Patommel, J., Seiboth, F., Lee, H. J., Nagler, B., Galtier, E. C., Arnold, B., Zastrau, U., Hastings, J. B., Nilsson, D., Uhlén, F., Vogt, U., Hertz, H. M. \& Schroer, C. G. (2013). Sci. Rep. 3, 1633.

Seaberg, M., Cojocaru, R., Berujon, S., Ziegler, E., Jaggi, A., Krempasky, J., Seiboth, F., Aquila, A., Liu, Y., Sakdinawat, A., Lee, H. J., Flechsig, U., Patthey, L., Koch, F., Seniutinas, G., David, C., Zhu, D., Mikeš, L., Makita, M., Koyama, T., Mancuso, A. P., Chapman, H. N. \& Vagovič, P. (2019). J. Synchrotron Rad. 26, 11151126.

Seiboth, F., Kahnt, M., Lyubomirskiy, M., Seyrich, M., Wittwer, F., Ullsperger, T., Nolte, S., Batey, D., Rau, C. \& Schroer, C. G. (2019). Opt. Lett. 44, 4622-4625.

Seiboth, F., Scholz, M., Patommel, J., Hoppe, R., Wittwer, F., Reinhardt, J., Seidel, J., Knaut, M., Jahn, A., Richter, K., Bartha, J. W., Falkenberg, G. \& Schroer, C. G. (2014a). Appl. Phys. Lett. $\mathbf{1 0 5}, 131110$.

Seiboth, F., Schropp, A., Hoppe, R., Meier, V., Patommel, J., Lee, H. J., Nagler, B., Galtier, E. C., Arnold, B., Zastrau, U., Hastings, J. B., Nilsson, D., Uhlén, F., Vogt, U., Hertz, H. M. \& Schroer, C. G. (2014b). J. Phys. Conf. Ser. 499, 012004.

Seiboth, F., Schropp, A., Scholz, M., Wittwer, F., Rödel, C., Wünsche, M., Ullsperger, T., Nolte, S., Rahomäki, J., Parfeniukas, K., Giakoumidis, S., Vogt, U., Wagner, U., Rau, C., Boesenberg, U., Garrevoet, J., Falkenberg, G., Galtier, E. C., Ja Lee, H., Nagler, B. \& Schroer, C. G. (2017). Nat. Commun. 8, 14623.

Seiboth, F., Wittwer, F., Scholz, M., Kahnt, M., Seyrich, M., Schropp, A., Wagner, U., Rau, C., Garrevoet, J., Falkenberg, G. \& Schroer, C. G. (2018). J. Synchrotron Rad. 25, 108-115.

Streun, A., Garvey, T., Rivkin, L., Schlott, V., Schmidt, T., Willmott, P. \& Wrulich, A. (2018). J. Synchrotron Rad. 25, 631-641. 
Tavares, P. F., Al-Dmour, E., Andersson, Å., Cullinan, F., Jensen, B. N., Olsson, D., Olsson, D. K., Sjöström, M., Tarawneh, H., Thorin, S. \& Vorozhtsov, A. (2018). J. Synchrotron Rad. 25, 1291-1316.

Terentyev, S., Polikarpov, M., Snigireva, I., Di Michiel, M., Zholudev, S., Yunkin, V., Kuznetsov, S., Blank, V. \& Snigirev, A. (2017). J. Synchrotron Rad. 24, 103-109.

Thibault, P., Dierolf, M., Menzel, A., Bunk, O., David, C. \& Pfeiffer, F. (2008). Science, 321, 379-382.

Uhlén, F., Nilsson, D., Holmberg, A., Hertz, H. M., Schroer, C. G., Seiboth, F., Patommel, J., Meier, V., Hoppe, R., Schropp, A., Lee,
H. J., Nagler, B., Galtier, E., Krzywinski, J., Sinn, H. \& Vogt, U. (2013). Opt. Express, 21, 8051-8061.

Uhlén, F., Nilsson, D., Rahomäki, J., Belova, L., Schroer, C. G., Seiboth, F., Holmberg, A., Hertz, H. M. \& Vogt, U. (2014). Microelectron. Eng. 116, 40-43.

Vila-Comamala, J., Diaz, A., Guizar-Sicairos, M., Mantion, A., Kewish, C. M., Menzel, A., Bunk, O. \& David, C. (2011). Opt. Express, 19, 21333-21344.

Vila-Comamala, J., Sakdinawat, A. \& Guizar-Sicairos, M. (2014). Opt. Lett. 39, 5281-5284. 